\section{Incidence of Uraemia}

SIR,-Dr. R. A. Branch and his colleagues (30 January, p. 249) in their analysis of the incidence of uraemia found "a remarkable preponderance of elderly patients" with renal failure. We presume we are correct in our assumption that the laboratory records used in this survey were those at Bridgend General Hospital. We therefore wonder whether these authors are aware of the fact that during the period in question (1966-8) a haematological survey of elderly patients was being conducted, included in which was the routine estimation of the blood urea in all patients admitted to the geriatric wards? Furthermore, we have reason to believe that the population served is significantly in excess of the quoted 120,000.-We are, etc.,

\section{E. B. POWELL}

J. H. Thomas

Pathology Department,

Bridgend Genera
Bridgend, Glam

\section{Haemophilus aphrophilus Endocarditis}

SIR,-From time to time you publish case reports of endocarditis due to Haemophilus aphrophilus. The article of Dr. R. M. Pine and Dr. H. S. Ballard (9 May, p. 344) stated that the organism they identified as $H$ aphrophilus did not have growth facto requirement other than carbon dioxide and that it fermented lactose and glucose with acid and gas formation. May I say that $H$. aphrophilus does not produce gas and that there was no mention of the 14 other "sugars" tested in my 1940 work. ${ }^{1}$ Also there is nothing strange in the murmurs being detected six weeks after admission, since the changes in the valves do not develop overnight. There are cases of endocarditis with murmurless valves. ${ }^{2}$

Being the discoverer of $H$. aphrophilus, when I isolated it from an endocarditis patient at University College Hospital London in $1938,{ }^{1}$ may I have a word on the subject?

For 25 years following the publication of $\mathrm{my}$ 1940 paper neither I nor anyone else isolated a second strain. It was a good thing deposited in 1939 six strains of my organism with the National Collection of Type Cultures. then at Chelsea. Then many papers appeared in the U.S.A. claiming the isolation of that organism from endocarditis, brain abscesses etc. As the author of the 1940 paper I could at once see that most of these writers had never read my nine-page original paper, although they mentioned it in their references. They simply read the half page about it published in the 1957 Bergey's Manual of Determinative Bacteriology. This was obvious in King and Tatum's paper. ${ }^{3}$ These two workers from the public health laboratory of the Communicable Disease Center of Atlanta, Georgia U.S.A., examined a variety of strains which they identified as $\boldsymbol{H}$. aphrophilus. Those strains were not $\mathrm{my}$ aphrophilus at all since they needed no $\mathrm{X}$ factor and no excess of $\mathrm{CO}_{2}$ and moreover produced gas in the fermentation tests. Page and King ${ }^{4}$ also claimed that $H$. aphrophilus needed neither $\mathrm{X}$ factor nor excess $\mathrm{CO}_{2}$, having examined old stock cultures.

In addition, all these workers used primitive techniques such as a candle jar, and other workers in England added marble chips to some acid in a test tube. Since 1940, whether in England, Africa (Egypt), or Canada, I have been incubating all my cultures from pathological material (blood cultures, pus, cerebrospinal fluid, urine, etc., except stools) on blood agar plates in triplicate, in air containing 5\% $\mathrm{CO}_{2}$ as well as aerobically and anaerobically.
The $\mathrm{CO}_{2}$ has been obtained either from a Kipp's generator or from cylinders, always using a one-limb mercury manometer. Even as ong ago as 1938 I tested $\mathrm{CO}_{2}$ atmospheres ranging from 0.1 to $90 \%$ in air. 1

Other workers used unwashed viable aphrophilus organisms, thus not eliminating a carry over of preformed $\mathrm{X}$ factor with the inoculum from the mother culture. Thrice washing in peptone water would avoid this, while meat extract (broth) contains heat-stable $X$ factor from the minute amounts of blood in the capillaries of the meat. That is why satellitism experiments should not be made on nutrien agar but on plain agar made with peptone, sodium chloride, and glass-distilled water. Without such precautions those who think thev could revolutionize the classification of the Haemophilus group should only blame themselves.

My 1940 paper contained a very accurate description of aphrophilus when freshly isolated, especially its need for $\mathrm{X}$ factor and excess $\mathrm{CO}_{2}$. Workers isolating organisms suspected of being $H$. aphrophilus should refer to my original description in the 1940 paper and not compare their organisms with 30-year-old stock cultures, kept under heaven knows what conditions, grown possibly on media deficient in X factor, and incubated in ordinary atmospheric air not containing excess $\mathrm{CO}_{2}$.

Another point is about $H$. gallinarum of Eliot and Lewis, ${ }^{6}$ which is stated on page 504 of my 1940 paper as being remarkably enhanced in a carbon dioxide atmosphere and requires $\mathrm{V}$ factor (not $\mathrm{X}$ ). The full reference is mentioned at the end of that paper. Yet someone in Leeds claimed in 1968 to have "discovered" a new species requiring $\mathrm{V}$ factor and excess $\mathrm{CO}_{2}$ and gave it the name $H$. paraaphrophilus, thus ignoring that Eliot and Lewis had already 34 years previously given the same organism the name $H$. gallinarum, which should have priority in nomenclature. To this may I draw the attention of editors of textbooks on bacteriology.

If all this happens to my $H$. aphrophilus while I am still alive and in full activity at the age of 61 , what will happen to it after I am dead? - I am, etc.,

OMar Khairat

Department of Medical Bacteriology,

University of Alexandria,

Khairat, Omar, fournal of Pathology and Bacteriology, 1940, 50, 497

MacGregor, G. A., Lancet, 1962, 2, 993.

King, Elizabeth O., and Tatum, H. W., fournal Page, M. I., and King, Elizabeth $O$., New Eng land Fournal of Medicine, 1966, 275, 181

ilson, Sir Graham, and Miles, Sir Ashley, opley and Wilson: Principles of Bacteriology Edward Arnold, 1964

6 Eliot, C. P., and Lewis, M. R., fournal of the American "Veterinary Medical Association, 1934,
84, 878.

\section{Torsion of the Testicle}

SIR,-For the fourth time in five years it has been my unhappy duty to excise a gangrenous testicle from a young man. These four patients, aged from nine to 28 years, had suffered torsion of the testicle which had been neglected. They all gave an identical history of testicular pain and swelling with associated inflammation of the scrotal skin and all had sought early medical advice. Antibiotics had been prescribed for their supposed epididymitis by either a general practitioner or a casualty officer.

Epididymitis in young males is extremely rare in the absence of urinary tract disorder. Any child or young man with testicular pain and swelling should be admitted to hospital as an emergency and his scrotum should be explored. This is a relatively minor pro- cedure which will prevent the unnecessary disaster of testicular gangrene due to torsion and will enable early diagnosis of the occasional testicular tumour which may present in this way. In the unlikely event that epididymitis is the cause of the trouble, no harm will be done by exposure of the testicle.

If torsion is found it is obligatory to expose the other testicle at some stage because the anatomical abnormality which permits torsion to occur is usually bilateral. It is probably unwise to do this at the same operation if the twisted testicle is gangrenous, as infection may follow, but if early operation has been performed the condition can be corrected and both testicles anchored to prevent recurrence-I am, etc., London Hospital,
London E.1

RICHARD G. NOTLEY

\section{Folic Acid in Epilepsy}

SIR,-Dr. A. Richens (9 January, p. 109) has drawn attention to some apparent deficiencies of our trial of folic acid in folatedeficient patients with epilepsy (12 December, p. 644).

Though the use of a $2 \times 2 \times \chi^{2}$ test might lead one to conclude that a significantly higher number of patients showed an increase in grand mal fits in the folic acid group compared to the placebo group ( $\mathbf{p}<0.5)$ this conclusicn would appear to be of doubtful validity. Thus if one considers the three categories, "increase," "decrease," and "no chang:-," a similar $\chi^{2}$ test reduces the significance level to $10 \%$. If the magnitude of the changes is considered then the "significance" of the differences disappears completely. If we consider only patients receiving folic acid before and after treatment, it is reasonable to suppose that if folic acid produced no effect on the frequency of grand mal we would expect half the patients to show an apparent increase and half to show a reduction. Reference to Table II shows that this is in fact what happened. Although having some sympathy with $\mathrm{Dr}$. Richens's remarks on the use of the " $t$ " test we would like to point out that this test was based on the differences in the number of attacks before and after treatment which ranged from -97 to +25 . Expressing the changes as a percentage of the pre-treatment level would have meant an infinite ratio for those patients wit! no fits in the pre-treatment period, which is not very meaningful in clinical terms, especially considering the natural variation of the condition.

With regard to the recording of fits we would submit that the accuracy is likely to be much higher in an institution such as this than in many other circumstances, especially outpatient assessment, and if there are inaccuracies then they will apply equally to both groups over a period as long as six months. Grand mal fits in particular are exceptionally unlikely to occur unrecorded.

Reynolds in his original work ${ }^{1}$ pointed out that the majority of patients did not show an increase in fit frequency until several months after starting treatment with folic acid. Our results for the period 27-52 weeks' treatment show no differences whatever in fit frequency between the folic acid and placebo groups.

Patients with serum phenytoin levels greater than $25 \mu \mathrm{g} / \mathrm{ml}$ were excluded or 